\title{
LA EXCLUSIÓN SOCIAL Y LAS TECNOLOGÍAS DE LA INFORMACIÓN Y LA COMUNICACIÓN: UNA VISIÓN ESTADÍSTICA DE SU RELACIÓN EN LA EDUCACIÓN SUPERIOR
}

\section{Social Exclusion and Information and Communication TeChnologies: A Statistical View of Their Relationship in Higher Education}

\author{
Martha Patricia Astudillo-Torres* \\ Florlenis Chévez-Ponce** \\ Yesenia Oviedo-Vargas***
}

DOI: http://dx.doi.org/10.29043/liminar.vl8il.721

\begin{abstract}
Resumen: En este artículo se expone el proceso de diseño e implementación de una escala inédita que mide la integración de las Tecnologías de la Información y la Comunicación (TIC) en las prácticas educativas universitarias y la generación de exclusión social derivada de esta integración tecnológica. La recopilación de información se realizó a través de una escala Likerth; el instrumento fue aplicado a estudiantes de dos centros de educación superior ubicados en México y Costa Rica. Los principales resultados señalan que el estudiantado valora las TIC como herramientas significativas en su formación; sin embargo, se excluye a estudiantes que no tienen un fuerte desempeño en estas tecnologías. Asimismo, de acuerdo con las evidencias empíricas generadas en el estudio se afirma que la escala contiene índices de consistencia interna y de confiabilidad apropiados para ser aplicada en la medición de los tópicos que se conforman para poblaciones de nivel educativo superior.
\end{abstract}

Palabras clave: exclusión social; inequidad; brecha digital; sociedad de la información; tecnologías de la información y la comunicación.

Abstract: This article discusses the design and the implementation of a scale that measures the integration of information and communication technologies (ICT) in university educational and the generation of social exclusion derived thereof. Information was gathered through a Likerth scale applied to students at two higher education centers in Mexico and Costa Rica. The main results indicate that students value ICT as significant tools in their education; however, students who do not perform well with these technologies are often excluded. Likewise, according to the empirical evidence generated in the study, we conclude that the scale contains indexes of internal consistency and reliability such that it can be applied in measuring populations at higher educational centers.

Keywords: social exclusion; inequity; digital divide; society of information; information and communications technology.

\footnotetext{
* Martha Patricia Astudillo Torres. Doctora en Educación por la Universidad de Costa Rica. Profesora-investigadora en la Universidad Autónoma de Chiapas, México. Temas de especialización: tecnologías de la información y la comunicación en educación superior, desigualdades sociales en el entorno educativo, diseños metodológicos con perspectiva positivista e interpretativa; evaluación educativa. Correo electrónico: patricia.astudillo@ unach.mx. ORCID: https://orcid.org/0000-0002-9013-6467.

** Florlenis Chévez Ponce. Doctora en Educación por la Universidad de Costa Rica. Profesora-investigadora en la Facultad de Educación de la Universidad de Costa Rica. Temas de especialización: desigualdades sociales en el contexto de educación básica, media y superior, evaluación
}

educativa. Correo electrónico: florlenis.chevez.ponce@mep.go.cr. ORCID: https://orcid.org/0000-0002-6295-9052.

*** Yesenia Oviedo Vargas. Doctoranda en Educación por la Universidad de Costa Rica. Profesora-investigadora en la Facultad de Educación de la Universidad de Costa Rica. Temas de especialización: evaluación educativa, factores asociados al rendimiento académico. Correo electrónico: yesenia. oviedovargas@ucr.ac.cr; yessenia.oviedo.vargas@mep.go.cr ORCID: https:// orcid.org/0000-0002-4079-6029.

Enviado a dictamen: 27 de mayo de 2019

Aprobación: 22 de octubre de 2019.

Revisiones: 2 


\section{Introducción}

1 partir de la incorporación creciente al contexto social de las Tecnologías de la Información y la Comunicación (TIC) en la última década se han acuñado nuevos términos relacionados con la integración de dichas tecnologías a diversos ámbitos colectivos; uno de estos términos es el de brecha digital. De acuerdo con Alva (2015), la brecha digital representa una nueva expresión de la desigualdad en el siglo XXI y conlleva la marginación de amplios sectores sociales en el acceso, el uso y la apropiación de los bienes y servicios de las telecomunicaciones y las TIC, que permiten a las personas participar o no en el desarrollo de la nueva sociedad que se construye.

De igual forma, la brecha digital constituye un problema para el desarrollo social debido a que, en la medida en que la población no accede equitativamente a las TIC, emerge una nueva forma de exclusión social, de manera que ciertos sectores de la población quedan marginados de las ventajas que genera el uso de las TIC, tales como las oportunidades de empleo, la interacción y la integración social (CEPAL, 2010). Además, el término se relaciona con la desigualdad en capacidades y habilidades de los individuos para participar y desarrollarse en las "sociedades de la información y el conocimiento" (Castaño, 2008).

En este sentido, las autoras de este trabajo coinciden con la perspectiva según la cual la conectividad es importante, pero no suficiente para contribuir al desarrollo de la sociedad de los países emergentes puesto que es necesario, además, aprovechar las oportunidades y los posibles resultados positivos que proporcionan el acceso equitativo, el uso con sentido y la apropiación social de los recursos de las TIC.

Con tal base, el objetivo del este estudio radica en el análisis de la relación entre la exclusión social y el uso de las TIC en la educación superior en dos casos concretos: la Universidad Autónoma de Chiapas, en México, y la Universidad de Costa Rica. Asimismo, la hipótesis que se plantea es que uno de los aspectos de exclusión social en los contextos universitarios está asociado al uso de las TIC.
A partir del planteamiento de la hipótesis surgen dos categorías que permiten un análisis de la relación expuesta. La primera categoría es la exclusión social definida desde lo que plantea Dubet (2009), quien señala que es un problema social en el que la escuela ratifica los fenómenos de exclusión. En relación con este planteamiento, considera que, en el avance tecnológico en la sociedad, "la exclusión social aparece para aprehender y dar sentido teórico a esas nuevas lógicas desigualitarias inherentes a ese desarrollo plagado de abundantes referencias a procesos excluyentes" (Tezanos, 2001:30). Por tanto, esta categoría genera elementos que permiten entender la relación que se forma en el actuar al establecer carencias que sitúan a las personas en posiciones de desventaja social en diferentes ámbitos: económicos, políticos, culturales, educativos y sociales en general.

Respecto a la segunda categoría, la integración de las TIC en la educación, esta se retoma desde los señalamientos establecidos por las políticas educativas en el panorama mundial, regional, nacional y local, las cuales manifiestan la importancia de incluir las TIC en todos los niveles educativos para la mejora del proceso de enseñanza y aprendizaje (Gobierno del Estado de Chiapas, 2019; Presidencia de la República, 2019; UNACH, 2019; UNESCO, 2005, 2007 y 2008). Sin embargo, en estos planteamientos no se establece la forma en que dichas tecnologías deben ser incorporadas a los contenidos curriculares de las diversas asignaturas para mejorar el proceso educativo.

En el caso de México, la inequidad presenta varias facetas y expresiones, a las que se suma la brecha digital, la cual ha sido abordada desde múltiples miradas en investigaciones recientes (Alcalá, 2019; Campuzano, 2019; Gómez, 2019; Gómez et al., 2018; Toudert, 2019); no obstante, en este estudio se pretende analizar la relación entre los ejes expuestos (exclusión social y TIC en la educación universitaria) desde la perspectiva positivista, realizando una medición estadística para explicar la asociación que se suscita desde estos dos ámbitos en la educación superior.

Respecto a Costa Rica, actualmente se encuentran situaciones que excluyen a diversos grupos, por lo que se requieren políticas a favor de los grupos discriminados y 
excluidos. Esto se refleja en una distribución inequitativa en la que "los recursos y oportunidades provocan que grupos de la población enfrenten privaciones múltiples - por ejemplo, la falta de acceso a servicios básicos y a oportunidades necesarias como la educación" (León y Mata, 2017:16). Por lo expuesto, se justifica la importancia de la realización de investigaciones de este tipo en los países en referencia.

\section{Marco teórico}

La exclusión debe considerarse como un proceso en el cual se presentan distintos estadios que culmina con una completa postergación, por lo que es evidente que existe un contexto en el que la exclusión social se presenta cada vez con mayor protagonismo en los campos educativo, político, académico y social. Al respecto, se establece que uno de los logros importantes del enfoque teórico de la exclusión social es que se obliga a "centrar el debate en aspectos que la investigación tradicional de la pobreza había dejado al margen, como la importancia de las relaciones sociales, la multidimensionalidad del fenómeno o la necesidad de estudiar las dinámicas que conducen a la exclusión" (Jiménez, Luengo y Taberner, 2008:20).

En cuanto a la exclusión social, como fenómeno inscrito en actos y decisiones de agentes, como lo son las instituciones educativas, las organizaciones sindicales y la familia, entre otras, se instaura en una "clara dimensión estructural la cual debe articularse con su naturaleza relativa y enmarcada en una red de agentes que adoptan decisiones de las que pueden derivar procesos de exclusión, de manera que la exclusión social expresa la nueva configuración de las desigualdades en el contexto actual" (Subirats, Gomà y Brugué, 2005:11). Por ello, en un sistema educativo donde la misma educación pública está fracturada y se estimula la educación privada como mejor opción, se establece una distinción entre estudiantes que asisten a una u otra escuela, lo que de algún modo propicia tempranamente la exclusión social desde el hecho de ser parte de un tipo de educación particular (OREAL/ UNESCO, 2011).
Los objetivos de la educación están planteados para construir espacios que permitan el desenvolvimiento social y las interacciones personales, mediante criterios que sean consensuados y que defiendan la equidad escolar y la participación estudiantil activa. Sin embargo, en la educación se establece una contradicción en relación con los procesos de exclusión, ya que la misma escuela es potenciadora de esta al dejar de lado su función social de ser un espacio donde se genere integración. Por tanto, este contexto educativo "introduce un verdadero cambio sobre el paradigma de la exclusión, ya que la paradoja de la escuela de la igualdad de oportunidades obedece a que sus ambiciones y su apertura hacen que ella misma se convierta en agente de exclusión" (Dubet, 2005:97). No obstante, es necesario señalar que la exclusión abarca:

Prácticamente todas las dimensiones de la personalidad de los estudiantes (intelectuales, personales y emotivas, sociales y actitudinales...) e incide en instancias y sujetos que están a su alrededor. Asimismo, resulta de la confluencia e interacción de elementos, estructuras y dinámicas diferentes, situadas, además, en diversos niveles (familias y entornos de socialización de los estudiantes, relaciones con el grupo de iguales, organización y gestión de los centros escolares y seguramente redes de centros, además del tipo de relaciones y alianzas entre cada centro y su medio, así como el currículo, la enseñanza y evaluación, la consideración y la formación del profesorado, los sistemas y dinámicas de asesoramiento escolar, desarrollo de los centros y el profesorado (Escudero, 2005:14).

Para efectos de esta investigación se considera el posicionamiento de Johnson y Rudolph (2001), quienes exponen que la exclusión social se establece desde dos niveles: el meso nivel, en el que se encuentran las decisiones que generan las políticas de gobierno, y el nivel micro, en el que se considera el espacio del aula donde se desarrolla el currículo.

Asimismo, la exclusión implica fracturas en el tejido social, la ruptura de ciertas coordenadas básicas 
de integración y, en consecuencia, la aparición de una nueva escisión social en términos de dentro/fuera. Genera, por tanto, un nuevo sociograma de colectivos excluidos, es decir, "la exclusión es susceptible de ser abordada desde los valores, desde la acción colectiva, desde la práctica institucional y desde las políticas públicas" (Subirats, Gomà y Brugué, 2005:13).

Por consiguiente, la exclusión social se puede analizar y entender como un proceso multidimensional con dos componentes fundamentales: por un lado, la separación de personas individualmente, así como de colectivos, de los derechos sociales y, por otro lado, la evidencia de personas con acumulación de acceso que excluyen a personas que no corresponden a este grupo. Al respecto, se puede señalar que la exclusión social puede ser entendida como: "Una acumulación de procesos confluyentes con rupturas sucesivas que, arrancando del corazón de la economía, la política y la sociedad, van alejando e 'inferiorizando' a personas, grupos, comunidades y territorios con respecto a los centros de poder, los recursos y los valores dominantes" (Estevill, 2003:20, traducción propia).

Según Castel (1995), la exclusión no es un estado, sino que pasa por un recorrido y deja marca en el transitar de una zona de vulnerabilidad a una zona de ausencia, hasta llegar al aislamiento. Es decir, las personas pasan por un proceso en el que poco a poco van experimentando la pérdida de pertenecer a un grupo. Por ello, plantea que se pueden distinguir tres zonas de organización y cohesión social: integración, vulnerabilidad y exclusión, las cuales se presentan en la Figura 1 (ver Figura 1).

La exclusión refiere a diferentes elementos que limitan las oportunidades y que responden a situaciones particulares desde lo social, lo político, lo económico y lo educativo, entre otros elementos que afectan a diversos grupos en las interacciones de comunicación que se generan en los diferentes espacios de la sociedad, lo cual no escapa a lo que ocurre en el aula. En la Tabla 1 se establecen las esferas en las que se visualiza la exclusión (ver Tabla 1).

El planteamiento de la exclusión requiere considerar las características principales inmersas en este proceso, ello para clarificar cómo esas características impactan en mayor medida a las poblaciones más vulnerables. Las características de la exclusión social se reflejan en la Tabla 2 (ver Tabla 2).

La exclusión educativa tiene que ver con el no acceso a los elementos indispensables para participar en procesos académicos; para efectos de esta investigación se relaciona con el no acceso al uso de las TIC y la exclusión del conocimiento. En este sentido, la política educativa tiene que dirigirse a la resolución de estos dos aspectos al mismo tiempo: por una parte, se deben implementar posibilidades para incorporar a los sujetos excluidos de la escolarización y, por otra parte, se deben brindar las herramientas para que los que estén sigan siendo parte del contexto educativo. De acuerdo con Dubet (2005), los procesos de exclusión se han incorporado a la escuela de forma progresiva, de manera que la población estudiantil no cuenta con las mismas oportunidades que permitirían el logro de un desarrollo educativo homogéneo.

De acuerdo con Ampudia y Delgado (2011), la desigual distribución de bienes, de servicios y de conocimiento entre los países no ha disminuido pese a la evolución social y tecnológica. Derivado de ello, la marginación continúa y al mismo tiempo aparecen nuevas formas de diferenciación social.

En este aspecto, una forma de exclusión en el siglo XXI es la brecha digital, surgida en el marco del modelo económico del capitalismo global, que consiste en las inequidades entre los grupos sociales en términos del acceso, las diferencias cognitivas, el conocimiento o las competencias para el uso de las TIC; igualmente, la brecha digital refiere a las discrepancias en las condiciones institucionales que permiten a algunos desarrollarse y participar en la sociedad de la información y el conocimiento, o bien, que no estén siendo integrados a ella en función de su edad, género o etnia (Alva, 2015).

Sin embargo, de acuerdo con Volkow, dicha brecha puede acotarse atendiendo cuatro dimensiones: infraestructura, habilidades de uso, oferta deinformación y cambio cultural; en cada una de ellas es imprescindible plantear soluciones con métodos, técnicas, lenguajes o 
contenidos distintos (Volkow, 2003). La infraestructura es un elemento que conforma la brecha digital de carácter económico; este aspecto es prioritario debido a que la falta de recursos para adquirir una computadora o acceder a Internet acelera la exclusión social. En cuanto a las habilidades de uso, refieren a la necesidad de adquirir una formación mínima en el ámbito de las TIC y de la tecnología educativa para modernizar los procesos formativos con el apoyo de plataformas tecnológicas. En este sentido, conforme se avanza en la capacitación y actualización docente, se abren las perspectivas para generar contenidos y ofertas educativas que tomen en cuenta los diversos estilos de aprendizaje (Ampudia y Delgado, 2011:38-39), para, finalmente, considerar el cambio cultural consistente en el resultado de un proceso continuo y discontinuo enfocado a consolidar una cultura digital.

Por otro lado, literatura especializada (Urresti, 2008; Benítez, 2010; Benítez et al., 201l y 2013) evidencia que hay una interrelación entre la clase social y la disposición de los jóvenes hacia las TIC. Así, los jóvenes de sectores medios y altos que nacieron en hogares profusamente provistos con tecnología y conectividad, cuyos padres ya fueron usuarios de la computadora e Internet, suelen contar desde edades tempranas con computadoras personales. Los jóvenes de sectores populares, por el contrario, tienden a presentar un primer contacto tardío con la computadora e Internet, realizado en espacios de acceso público como la escuela o un cibercafé, y sus padres por lo general tienen poca o ninguna alfabetización digital.

De este modo, los estudios demuestran, en primer plano, que la condición socioeconómica de los individuos es un factor preponderante para acercar o alejar la brecha digital mediante la adquisición (o no adquisición) de las competencias informacionales que se producen gracias al acceso a los medios telemáticos y a las herramientas TIC.

Congruentecon lo expuesto, en elinformePerspectivas económicas de América Latina 2017: juventud, competencias y emprendimiento (OCDE/CEPAL/CAF, 2016) se señala que las actitudes de los padres hacia las TIC y su situación socioeconómica influyen no sólo en el acceso a las TIC por parte de los jóvenes, sino también en el modo en que estos usan los dispositivos tecnológicos, ya sea para tareas escolares o para otro tipo de usos.

Igualmente, en otros estudios (Urresti, 2008; Benítez, 2010; Benítez et al. 2011 y 2013) se señala que existe una visión optimista, de manera que el acceso y el aprovechamiento de las potencialidades habilitadas por las TIC puede permitir reducir las inequidades existentes, tanto entre países como entre grupos sociales, al poner al alcance de los más relegados recursos hasta ahora vedados y exclusivos para una elite económica y cultural.

\section{Diseño metodológico}

Se utilizó un enfoque cuantitativo, pero exploratorio, ya que una comparación entre países como lo son México, contextualizado en la Universidad Autónoma de Chiapas, y Costa Rica, analizando un caso en la Universidad de Costa Rica, es una temática novedosa. Se utilizó una escala Likert elaborada para indagar sobre la relación entre el uso de las TIC en la formación universitaria y la exclusión social del estudiantado. Con esta investigación se pretende generar evidencias, por primera vez, en el marco mencionado bajo una mirada teórica, por lo que no se pretende generalizar los resultados a otros centros de educación superior, sino más bien instar a la réplica en otros contextos para comparar resultados desde la práctica.

\section{Muestra}

La selección de la muestra fue simple al azar. En ella se consideró a estudiantes de la Facultad de Educación, de la Escuela de Formación Docente de la Universidad de Costa Rica, y a estudiantes de la Facultad de Contaduría y Administración del área de computación de la Universidad Autónoma de Chiapas, México. En ambas universidades se explicó detalladamente el contenido de los ítems que conformaban la escala a los estudiantes participantes de investigación, todos ellos mayores de edad, por lo cual no fue necesario solicitar permiso a los padres o tutores. Al trabajar con la aplicación del 
instrumento, los docentes conocían el propósito de la investigación y de la colaboración tanto suya como de los estudiantes que incluían la muestra.

La muestra de la Universidad de Costa Rica se conformó por 104 alumnos de distintas especializaciones y niveles en las carreras de la Facultad de Educación. El padrón de la universidad presentaba una población de 1500 estudiantes; mediante el uso del programa Excel se numeró a todos ellos y se hizo una especie de lotería, de tal forma que cada estudiante tuviera la misma probabilidad de salir seleccionado. Cabe destacar que ese muestreo se hizo sin reemplazo; sin embargo, todos los sujetos de estudio fueron localizados y aceptaron participar. Dicha muestra corresponde a estudiantes que provienen de diversos contextos geográficos y cursan distintas carreras relacionadas con la enseñanza, tanto en educación primaria como en secundaria. Se realizó con un nivel de confianza del $70 \%$ y un error del $2 \%$.

Por otra parte, la muestra de la Universidad Autónoma de Chiapas se conformó por 101 estudiantes de diversos niveles de la Facultad de Contaduría y Administración, específicamente de la Licenciatura en Sistemas Computacionales y de la Licenciatura en Ingeniería en Desarrollo y Tecnología de Software. En este caso, la población era de 530 y se procedió de igual forma que en el muestreo para Costa Rica. Los estudiantes de la Universidad Autónoma de Chiapas, en su mayoría, procedían de distintos municipios del estado de Chiapas y una minoría del vecino estado de Oaxaca.

Se especificó un nivel de confianza del 99\% y un error de medición del 1.5\%. El total de la muestra fue de 205 estudiantes de estos dos centros educativos de educación superior.

\section{Instrumento}

El instrumento utilizado fue una escala de opinión constituida en dos partes: la primera presenta datos generales de carácter sociodemográficos, y la segunda está conformada por reactivos dirigidos a los estudiantes cuyas temáticas versan sobre las TIC y la exclusión educativa. Se trata de una escala de 23 ítems tipo Likert de grado de acuerdo (cuatro grados de acuerdo).

Para la validación de la escala se estableció un proceso por jueceo en las áreas de medición y TIC, con la finalidad de determinar si los ítems presentaban relación directa con lo que se estudia. En este sentido, se presentó una determinación de considerar puntaje de l a -l. Así, el puntaje de l se interpretó en el sentido de que elítem es aceptado completamente, 0 tiene relación pero necesita mejoras y -1 no cumple con los requisitos mínimos para ser aceptado. Una vez incorporados los cambios se procedió a la aplicación de la escala.

\section{Procedimiento de aplicación}

El proceso de aplicación de la escala se realizó según la muestra establecida en cada uno de los centros educativos considerados para este estudio y se aplicó en forma personal por las investigadoras, de manera que la información obtenida en la aplicación piloto determina la versión final de la escala para una posterior aplicación en la última etapa de la investigación. El período de aplicación fue de dos meses, ya que se requería hacer visitas a las aulas en que se encontraban los estudiantes pertenecientes a la muestra. El uso de la escala puede ser replicable en términos que se puede ampliar a más centros educativos.

\section{Resultados}

Los resultados parciales brindan evidencias acerca de la validez y confiabilidad del instrumento denominado "Escala: las desigualdades y las TIC en la educación superior". De acuerdo con Cronbach (1951), la consistencia interna de un instrumento se basa en una medida que está en relación directa con la confiabilidad y en relación indirecta con respecto a la validez del constructo que se mide, es decir, se evidencia una estimación en la correlación entre los ítems que conforman la escala.

El análisis se realizó con la ayuda del paquete estadístico SPSS versión 25; para ello, se efectuó un análisis factorial y un estudio de fiabilidad que permite 
estudiar la configuración de las dimensiones de la escala desde un punto de vista psicométrico y comparar con los referentes teóricos en los cuales se basa la investigación.

Se presenta, en primer lugar, el estudio de las comunalidades donde el parámetro que se toma es superior al 50\%, es decir, 0.500, el cual devela que los reactivos configuran bien. Para efectos de esta investigación se establece como una comunalidad moderada un parámetro superior a 0.300. Es importante aclarar que comunalidades muy bajas pueden indicar que los reactivos no carguen en el componente previsto.

El análisis factorial exploratorio de la escala aplicada en México revela que las comunalidades de los reactivos 4, 6 y 7 podrían generar problemas al momento de cargar en algún componente, mientras que un total de 11 reactivos de la escala presentan comunalidades mayores al 30\%. Los demás oscilan entre el 20\% y el 30\%. En el caso del reactivo 19 presenta parámetros de comunalidades óptimos. La Tabla 3 presenta dichos resultados (ver Tabla 3).

Por otro lado, en Costa Rica los datos se comportan diferente. En la Tabla 3 se muestra que todos los reactivos presentan comunalidades mayores al 30\% (ver Tabla 3).

Los datos que presenta la varianza explicada para México se exponen en la Tabla 4, en la que se muestra que, en este país, el primer componente o factor muestra corresponde a un porcentaje del $21 \%$. Teóricamente el primer componente debe ser igual o mayor que el $10 \%$, por lo que se cumple para este caso. Sin embargo, el segundo componente debe ser menor que un $10 \%$ y aquí, lo supera; los demás componentes presentan un valor inferior al 10\% (ver Tabla 4).

Los datos que presenta la varianza explicada para Costa Rica se exponen en la Tabla 5, en la que se muestra que en el caso de este país los primeros 13 componentes presentan un valor mayor que el $2 \%$. Mientras que el primer componente muestra un porcentaje bastante alto, del 34,64\% lo cual teóricamente es un porcentaje ideal, ya que el segundo componente no supera el 10\% (ver Tabla 5).

El análisis exploratorio, desde la información que se genera en los gráficos de sedimentación de Catell, proporciona evidencia de que para el caso de México se configuran al menos dos dimensiones (ver Figura 2), mientras que en el caso de Costa Rica, estos datos evidencian que se configura fuertemente en una sola dimensión (ver Figura 3).

A partir de las dimensiones que se encuentran en México, se realizó un segundo estudio factorial para obtener los reactivos que configurarán en dos componentes y analizar cuales forman parte de esta configuración, tal como se visualiza en la Tabla 6 (ver Tabla 6).

Según los datos de la Tabla 6, para el caso de México se configuran dos grandes dimensiones. Se toman como cargas factoriales aquellos reactivos que muestran datos mayores que 0.30 . Existe un primer componente con los reactivos 3,5,7,8,9,10,11,12.15,18,19,21 y 23. Un segundo componente se configura con los reactivos 1 , 2,13.14, 16, 20 y 22. Por otra parte, la correlación de esos dos componentes es moderada de 290 (ver Tabla 6).

Se realizó un estudio de fiabilidad para cada componente, lo que resultó que en el primer factor la confiabilidad es bastante buena, de 0.80 . Los reactivos resultantes en su mayoría presentan discriminaciones superiores a 0.30 y no es necesario eliminar un reactivo, ya que no se contribuye a elevar el Alfa.

En el segundo factor, luego de eliminar el reactivo indicado como "La utilización de las TIC ayuda a un mejor desarrollo de los contenidos impartidos por sus docentes" se obtuvo un alfa de apenas un 0.631 y 6 elementos, por lo que se muestra un parámetro de confiabilidad débil. Sin embargo, como se observa en la tabla, los demás reactivos presentan discriminaciones bastantes altas (o sea mayor que 0.30 )

En el caso de Costa Rica se procedió a realizar el estudio de fiabilidad, ya que sí se configuró una sola dimensión en la escala. En primer lugar, se presenta la consistencia interna que se tuvo en Costa Rica: para los 23 ítems se obtuvo un Alfa de Cronbach de .896.

A partir del Alfa de Cronbach obtenido se puede señalar que existe una alta consistencia interna, al aplicar la escala con 23 ítems, lo que indica que hay evidencia de que los componentes que conforman el instrumento tienen una estrecha relación entre los ítems. 
De acuerdo con los datos, si se elimina el elemento o reactivo 13, se podría mejorar el Alfa de Cronbach en céntimas, por lo cual se tomó la decisión para el análisis de eliminar el ítem 13, ya que era el que más aportaba para la aplicación de Costa Rica.

Al realizar de nuevo el cálculo del Alfa de Cronbach se obtuvo un Alfa de 90.5, lo cual es un parámetro considerado alto y da evidencia de una alta consistencia interna del instrumento y su unidimensionalidad. Asimismo, existen reactivos con discriminaciones menores a 0.10 , pero es importante considerar que, aun si se le eliminan estos, no contribuyen en el aumento del Alfa y se debe tener presente que es una población menor que 100, por lo que se tomó la decisión de mantener la escala con 22 reactivos para la aplicación definitiva.

\section{Análisis de resultados}

En cuanto a los resultados que arroja el análisis de los ítems que conforman la escala, es preciso señalar que en su conjunto, y por el Alfa de Cronbach que se obtiene, la consistencia interna muestra que los estudiantes dan un valor relevante a las TIC en relación con su formación académica y, además, permite señalar que aquellos compañeros de grupo que no hacen un uso adecuado o no tienen fortalezas al utilizar las TIC pueden ser excluidos; de esta manera, la escala permite el análisis de la relación entre TIC y exclusión educativa. Este resultado justifica la importancia de la realización de investigaciones de esta naturaleza, al exponer que la tecnología es un elemento significativo en las prácticas educativas de estudiantes universitarios vulnerables, sobre todo al hablar de la población perteneciente a dos de los estados más pobres y rezagados de México: Chiapas y Oaxaca.

Dados los resultados en términos de análisis de las escalas, se considera que para la aplicación definitiva del instrumento, en el caso de México, se debe presentar en dos dimensiones, es decir, en dos escalas separadas, y lograr hacer inferencias con mayor precisión de la escala de mayor consistencia interna. Por otra parte, para el caso de Costa Rica la escala debe contemplar la última versión que da el Alfa más alto, y debido a que sí configura su unidimensionalidad, los resultados posteriores también se darán a la luz de un instrumento mucho más robusto.

En síntesis, se puede concluir que la escala denominada "las desigualdades y las TIC en la educación superior" es un instrumento que tiene índices apropiados, ya que presenta consistencia interna y confiabilidad aceptada para ser aplicada en la medición de los tópicos que se conforman para las poblaciones de nivel universitario. Asimismo, se recomienda el abordaje de los resultados encontrados para replicar el resultado en otras carreras de estudio y en otras instituciones de educación superior.

\section{Conclusión}

En la educación superior es de suma relevancia realizar investigaciones que permitan contar con evidencias empíricas de la integración de las TIC relacionadas con la exclusión que puede darse en el estudiantado a partir de su incorporación en el proceso de enseñanza y aprendizaje. En la actualidad, el uso de las tecnologías es indispensable, ya que se requieren en el día a día para la formación de profesionales, de manera que las TIC se deben convertir en herramientas que aporten elementos para un futuro desarrollo de estudiantes como profesionales, y que el impacto no conlleve la exclusión de estos en este nivel de educación, sino que permita la apropiación y las comunicaciones pertinentes en el proceso de formación.

Los resultados obtenidos permiten señalar que surge evidencia empírica de cómo los aspectos teóricos que fundamentan el estudio se ven reflejados en los datos obtenidos. Asimismo, se rescata la validación del instrumento, ya que fue fundamental para mejorar los reactivos de la escala que se aplicó. Para el proceso de aplicación, se seleccionó una muestra de carácter representativo que permitió obtener los primeros resultados de la investigación. Los datos permiten establecer características diferenciadas en los países donde se implementó.

En primer lugar, se indica que para México surgen dos dimensiones con estadísticas psicométricas 
aceptadas para un estudio académico; en segundo lugar, para Costa Rica la escala inicial se conformó en una sola dimensión. Estos aspectos se retoman para una aplicación final, de manera que los datos posibles que se obtengan permitan señalar de manera confirmatoria que se consideren estas escalas como potenciadoras del establecimiento de la relación que exista entre la exclusión y las TIC.

Los ítems 1, 2,13.14, 16, 20 y 22 relacionados con la integración de las TIC en el proceso educativo de los estudiantes permiten valorar sus percepciones sobre el uso que hacen de las mismas para su inclusión o exclusión en el contexto académico, ya sea de forma presencial, virtual o mixta. En este sentido, se aborda el uso de la tecnología y la permanencia en los estudios superiores, las herramientas tecnológicas educativas empleadas, los materiales didácticos digitalizados que son utilizados en la formación, los artículos de investigación situados en bibliotecas y repositorios digitales, el trato igualitario entre estudiantes, etcétera, lo que hace posible indagar sobre el nivel de inclusión o exclusión que pueden brindar estas herramientas tecnológicas en el medio educativo.

Los trece ítems 3,5,7,8,9,10,11,12.15,18,19,21 y 23 de la escala están estrechamente relacionados con elementos de orden social que podrían generar procesos de exclusión en estudiantes universitarios, a partir de algún componente perteneciente a las TIC. Entre ellos el valor agregado que generan las tecnologías en las aulas y cómo pueden evitar la exclusión; la manera en que el uso de las TIC ayuda a estudiar temáticas de índole social considerando las necesidades educativas y sociales de los estudiantes, generando acciones que pueden repercutir positivamente y la relación de la exclusión social con el uso de las TIC, pues mediante estas se abren posibilidades para disminuir la desigualdad que se genera entre los estudiantes. Realmente se requiere que la exclusión de estudiantes no sea producto de la incursión y el acceso a las tecnologías; sin embargo, hay evidencias de que esto ocurre en el contexto mexicano.

Tras el análisis se determinó que, al conformarse dos dimensiones, la aplicación final puede brindar evidencias desde ambas dimensiones o desde cada una de las escalas según su configuración; de esta manera se puede establecer que la exclusión a partir de las TIC, ya sea desde la utilización o el acceso, es un elemento que debe ser considerado en las investigaciones educativas, de manera que, según lo que se indica en los ítems, se refuerce la carencia de estudiantes que no tienen fortaleza en alguno de los dos aspectos.

\section{Referencias}

Alcalá Casillas, Myriam G. (2019). "Desigualdad en el acceso a internet en México y la afectación en el ejercicio del derecho humano a la información". En Nuevo Derecho, 15(24), 55-70.

Alva de la Selva, Alma Rosa (2015). "Los nuevos rostros de la desigualdad en el siglo XXI: la brecha digital". En Revista Mexicana de Ciencias Políticas y Sociales, 60(223), 265- 285. Doi: http://dx.doi.org/10.1016/ S0185-1918(15)72138-0

Ampudia, Vicente y Lourdes Trinidad Delgado (2011). "Prácticas sociales y culturales con TIC en la universidad". En Reencuentro. Análisis de Problemas Universitarios, (62), 37-45. Disponible en: http://www. redalyc.org/articulo.oa?id=34021066005

Bel Adell, Carmen (2002). "Exclusión social: origen y características". Ponencia presentada en el congreso Formación Específica en Compensación Educativa e Intercultural para Agentes Educativos, Murcia, 2002. Disponible en: http://enxarxats.intersindical.org/nee/ CE_exclusio.pdf

Benítez Larghi, Sebastián (2010). Cazadores de e-topias. La lucha desigual por la apropiación de las TIC en las Organizaciones de Trabajadores Desocupados. Tesis de doctorado, Buenos Aires.

Benítez Larghi, Sebastián et al. (2011). "The Appropriation of Public Access to ICT by Urban Poor Youth in Argentina". En F. Proenza, R. Barrantes y H. Galperín (eds.), ICT \& Social Change. Impact of Public Access to Computers and the Internet. Cambridge: MIT Press.

Benítez Larghi, Sebastián et al. (2013). "TIC, clase social y género: la constitución de desigualdades sociales y digitales en las juventudes argentinas". Ponencia presentada en las X Jornadas de Sociología, 1 al 6 
de julio, Buenos Aires. Disponible en: http://www. memoria.fahce.unlp.edu.ar/trab_eventos

Campuzano Pérez, Gabriel (2019). "La brecha digital en educación media superior en escuelas rurales, México 2018". En Atlante: Cuadernos de Educación y Desarrollo, (febrero). Disponible en: https://www.eumed.net/ rev/atlante/2019/02/brecha-digital-educacion.html// hdl.handle.net/20.500.11763/atlante1902brechadigital-educacion

Castaño, Cecilia (2008). La segunda brecha digital. Madrid: Cátedra.

Castel, Robert (1995). "Les pièges de l'exclusion". En Lieu Social et Politiques, Revue Internationale d'Action Communautaire, 34, 13-21. Disponible en: https://doi. org/10.7202/005065ar

CEPAL (Comisión Económica para América Latina y el Caribe) (2010). Las TIC para el crecimiento y la igualdad: renovando las estrategias de la sociedad de la información. Chile: CEPAL.

Cronbach, Lee J. (1951). "Coefficient alpha and the internal structure of test". En Psychometrika, 16(297). Disponible en: https://doi.org/10.1007/BF02310555

Dubet, Frangois (2005). "Exclusión social, exclusión escolar". En Julián J. Luengo (comp.), Paradigmas de gobernacióny de exclusión socialen la educación. Fundamentos para el análisis de la discriminación escolar contemporánea. Barcelona/México: Ediciones Pomares.

Dubet, Frangois (2009). "Las paradojas de la integración escolar Espacios en Blanco". En Revista de Educación, 19, junio, 197-214. Buenos Aires: Universidad Nacional del Centro de la Provincia de Buenos Aires.

Escudero Muñoz, Juan M. (2005). "Fracaso escolar, exclusión educativa: ¿de qué se excluye y cómo?". En Profesorado. Revista de Curriculum y Formación del Profesorado, 9(1), 1-25. Disponible en: https://www. ugr.es/-recfpro/rev9lARTl

Estevill, Jordi (2003). Panorama de la lutte contre l'exclusion sociale. Concepts et stratégies. Ginebra: Bureau international du Travail. Disponible en: https://www. ilo.org/public/libdoc/ilo/2003/103B09_267_fren

Gobierno del Estado de Chiapas (2019). Plan Estatal de Desarrollo Chiapas 2019-2024. Tuxtla Gutiérrez: Gobierno del Estado. Disponible en: http:/wwww.
haciendachiapas.gob.mx/planeacion/Informacion/ PED/PED-2019.pdf

Gómez Navarro, Dulce Angélica (2019). "Uso de las tecnologías de la información y la comunicación por universitarios mayas en un contexto de brecha digital en México". En Región y Sociedad, 31, ell30-ell30.

Gómez Navarro, Dulce Angélica, Raúl Arturo Alvarado López, Marlen Martínez Domínguez y Christian Díaz de León Castañeda (2018). "La brecha digital una revisión conceptual y aportaciones metodológicas para su estudio en México". En Entreciencias: Diálogos en la Sociedad del Conocimiento, 6(16), 49-64.

Hernández Pedreño, Manuel (2010). "El estudio de la pobreza y la exclusión social. Aproximación cuantitativa y cualitativa". En Revista Interuniversitaria de Formación del Profesorado, 69, 25-46. Disponible en: https://dialnet.unirioja.es/servlet/autor?codigo=282674

Jiménez, Magdalena, Julián J. Luengo y José Taberner (2008). "Exclusión social y exclusión educativa como fracasos. Conceptos y líneas para su comprensión e investigación". En Revista Profesorado de Curriculum y Formación del Profesorado, 13(3). Disponible en: https:// www.researchgate.net/publication/45087638

Johnson, Debra y Angela Rudolph (2001). Beyond Social Promotion and Retention: Five Strategics to Help Students Suceed. Naperville, IL: Learning Point Associates. Disponible en: https://www.readingrockets.org/ article/beyond-social-promotion-and-retention-fivestrategies-help-students-succeed

León Espinoza, Diana y Gabriela Mata Marín (2017). La desigualdad en Costa Rica y el cumplimiento de la Agenda 2030 para el Desarrollo Sostenible. Costa Rica: PNUDCosta Rica/FLACSO. Disponible en: https://www. undp.org/content/dam/costa_rica/docs/undp_cr_ contribucion_debate_cr.pdf

OCDE/CEPAL/CAF (2016), Perspectivas económicas de América Latina 2017: juventud, competencias y emprendimiento. París: OECD. Disponible en: http:// dx.doi.org/10.1787/leo-2017-es

OREALC/UNESCO (2011). Progreso hacia educación para todos en América Latina y el Caribe: Encuentro Preparatorio Regional Naciones Unidas. Temas educativos centrales en América Latina y el Caribe. Santiago de Chile: 
UNESCO. Disponible en: http://www.unesco.org/ new/fileadmin/MULTIMEDIA/FIELD/Santiago/ pdf/progreso-Buenos-Aires.pdf

UNESCO (2005). Hacia las sociedades del conocimiento. Informe mundial de la UNESCO. París: UNESCO. Disponible en: http://unesdoc.unesco.org/images/0014/001419/141908s. pdf

UNESCO (2007). Educación de calidad para todos: un asunto de derechos humanos. Santiago de Chile: UNESCO.

UNESCO (2008). Situación educativa de América Latina y el Caribe: garantizando la educación de calidad para todos. Santiago de Chile: UNESCO.

Presidencia de la República (2019). Plan Nacional de Desarrollo 2019-2024. México. Disponible en: https:// www.gob.mx/cenace/acciones-y-programas/plannacional-de-desarrollo-2019-2024-195029

Subirats i Humet. Joan, Ricard Gomà Carmona y Joaquim Brugué Torruella (2005). Análisis de los factores de exclusión social. Catalunya: Fundación BBVA/Institut d'Estudis Autonomics/Generalitat de Catalunya.

Tezanos, José Félix (2001). La sociedad dividida. Estructuras de clase y desigualdades en las sociedades tecnológicas. Madrid: Biblioteca Nueva.

Toudert, Djamel (2019). "Brecha digital, uso frecuente y aprovechamiento de Internet en México". En Convergencia Revista de Ciencias Sociales, 26(79), 1-27. Disponible en: https://convergencia.uaemex.mx/ article/view/10332/9224

UNACH (Universidad Autónoma de Chiapas) (2019). Proyecto Académico 2018-2022. México: UNACH.

Urresti, Marcelo (2008). Ciberculturas juveniles. Los jóvenes, sus prácticas y representaciones en la era de Internet. Buenos Aires: La Crujía.

Volkow, Natalia (2003). "La brecha digital, un concepto social con cuatro dimensiones". En Boletín de Política Informática, 6, 1-5.

Figura 1. Zonas de organización y cohesión social
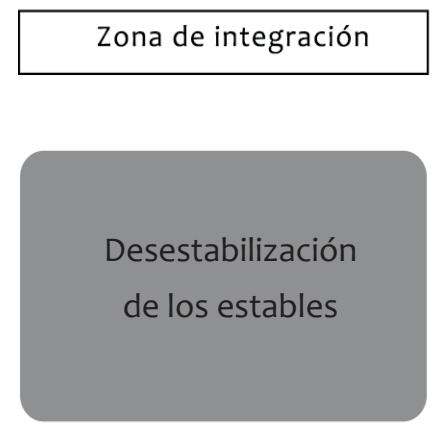
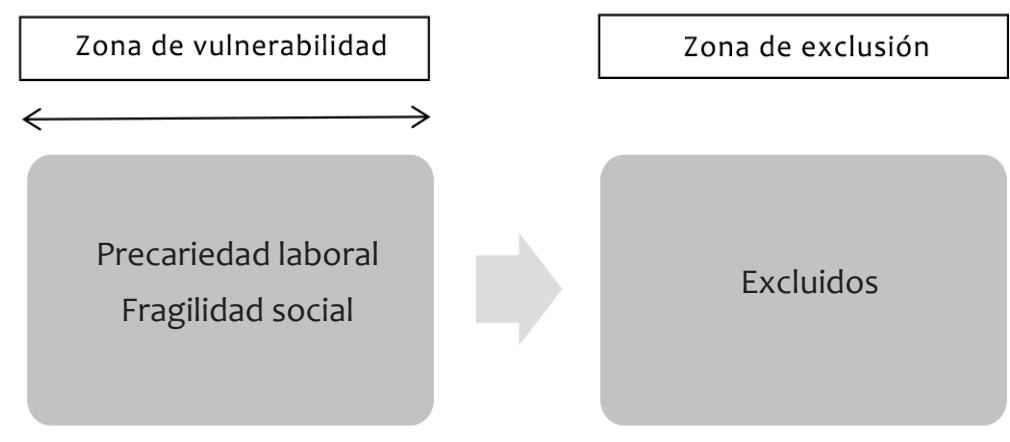

Fuente: elaboración propia (2019), con base en Castel (1995). 
Figura 2. Gráfico de sedimentación México

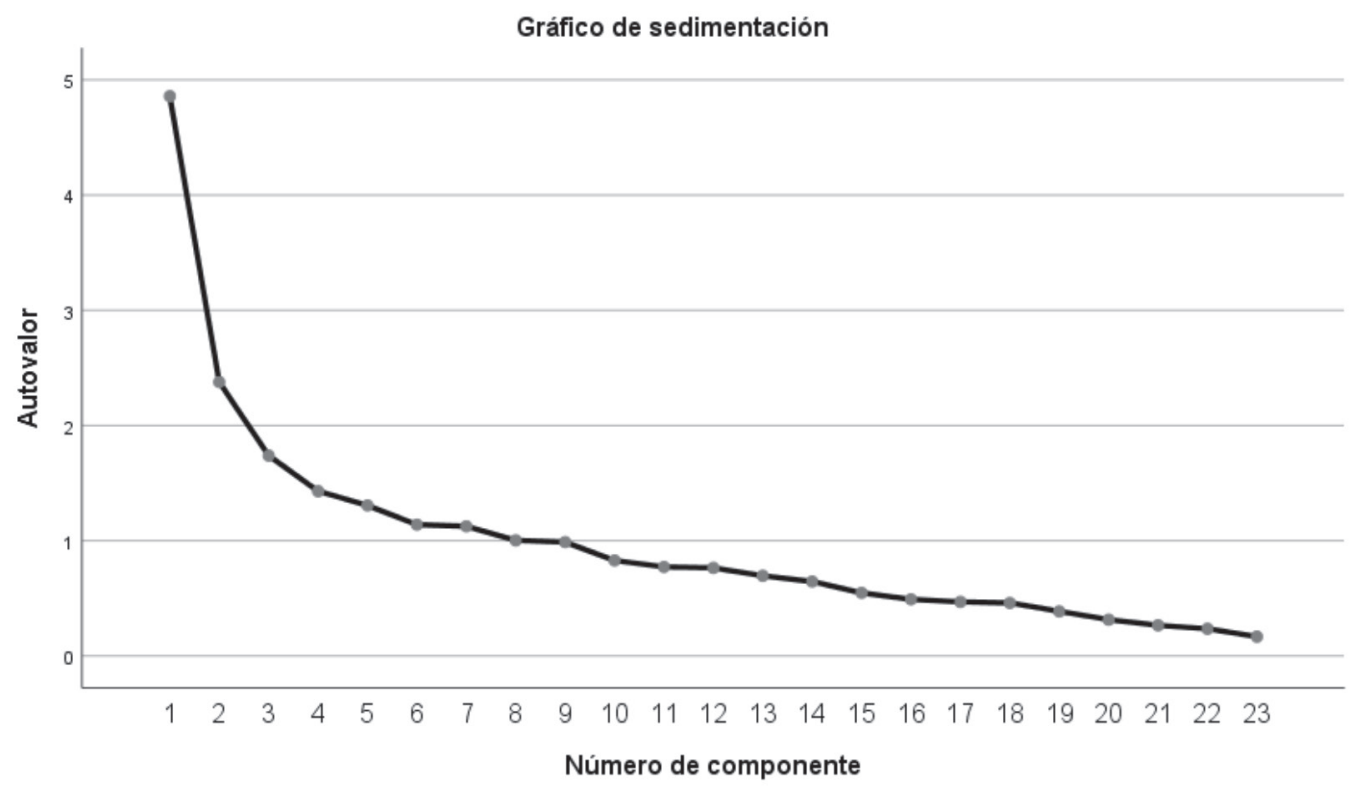

Fuente: Gráfico de Catell según análisis por software especializado. Escala de opinión relación opinión de estudiantes y utilización de las TIC. Construcción propia según análisis por software especializa.

Figura 3. Gráfico de sedimentación Costa Rica

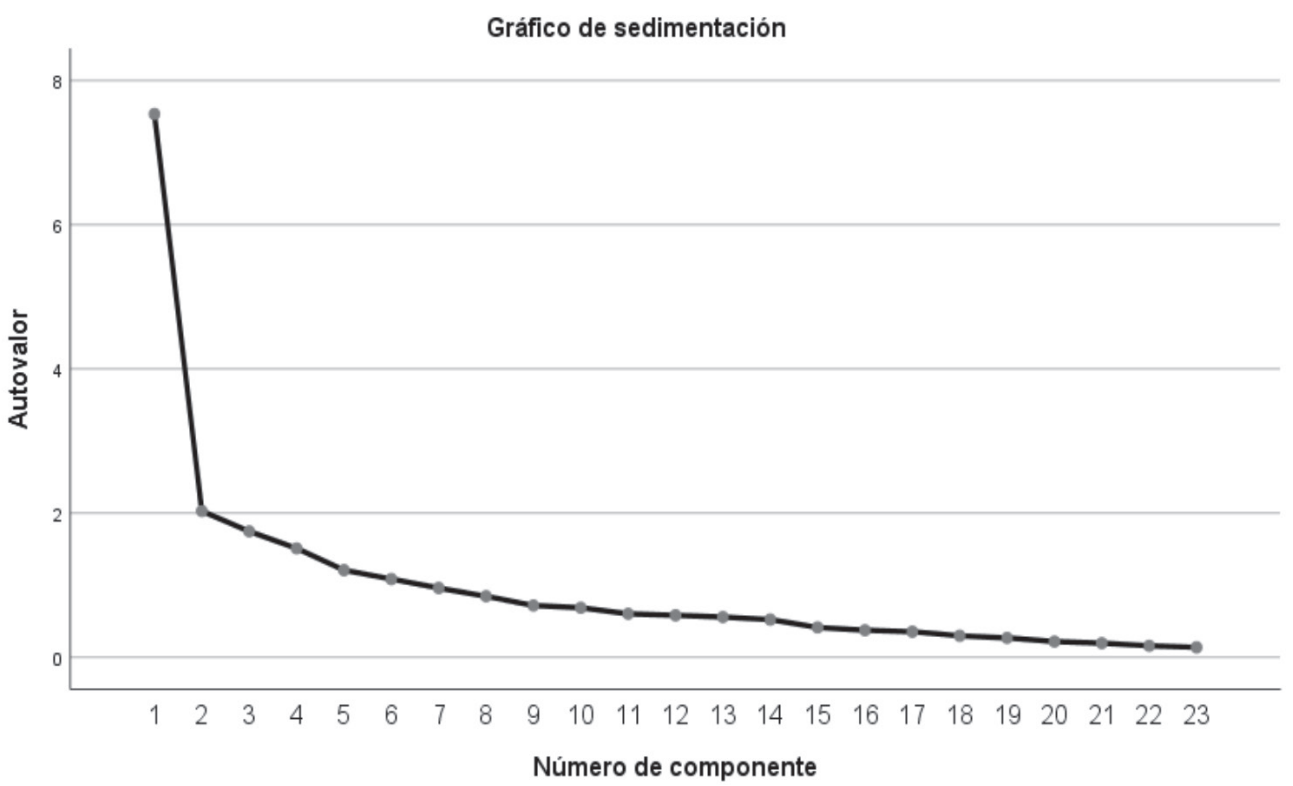

Fuente: Gráfico de Catell según análisis por software especializado. Escala de opinión relación opinión de estudiantes y utilización de las TIC. Construcción propia según análisis por software especializado (2019). 
Tabla 1. Esferas de la exclusión

\section{Elementos}

Colectivos y personas excluidas

Riesgo de "caer" en situaciones de exclusión

Carácter procesual y dinámico

Itinerarios de exclusión

Irreversibilidad e incapacidad del sujeto

Espacio de la exclusión: es al mismo tiempo homogéneo y heterogéneo

Mundo heterogéneo, complejo, incierto y desconcertante

Sujeto dispuesto a trabajar por su incorporación

\section{Descripción}

Presentan unas diferencias muy relevantes en cuanto a oportunidades vitales, condiciones y calidad de vida. Están al margen del modo de vida habitual en sociedades desarrolladas y siempre por debajo de un mínimo digno.

Está más extendido que nunca y puede llegar a afectar, y de hecho afecta, a personas y colectivos distintos de los pobres tradicionales y de los nuevos pobres.

La exclusión social no es tanto una situación (absoluta), sino un proceso de diversa intensidad según personas y grupos.

Son personales, familiares y, a veces, grupales.

Para combatir y salir de la exclusión, requiere apoyos externos y bien planificados.

El conocimiento del grado de la diversidad interna de los colectivos que lo integran, de sus posibilidades y limitaciones, necesidades y potencialidades es condición indispensable para una adecuada y efectiva intervención de incorporación diferenciada en sus contextos de referencia.

Ocupan una misma geografía: barrios, puerto, cárcel, calle, pero no hacen historia común, cada uno carga con su historia personal.

Conviene tenerlo en cuenta para no perder ese potencial, latente en muchos sujetos.

Fuente: Bel (2002).

Tabla 2. Características de la exclusión social

\begin{tabular}{lll}
\hline Es un fenómeno estructural & $\longrightarrow$ & $\begin{array}{l}\text { Deviene de causas estructurales y no meramente } \\
\text { individuales o causales. } \\
\text { Es un proceso } \\
\text { Muchas voces han destacado el carácter } \\
\text { dinámico y no estático de la exclusión. } \\
\text { multidimensional } \\
\text { Convergen múltiples factores interrelacionados } \\
\text { entre sí. } \\
\text { La multifactorialidad y la multidimensionalidad } \\
\text { de la exclusión conllevan a que sea un proceso } \\
\text { muy heterogéneo que afecta a muchos y } \\
\text { diversos grupos. }\end{array}$ \\
Es un fenómeno heterogéneo & $\longrightarrow \begin{array}{l}\text { Es susceptible de ser abordada con medidas } \\
\text { colectivas y desde la práctica institucional. }\end{array}$ \\
$\begin{array}{l}\text { Es abordable desde las políticas } \\
\text { públicas }\end{array}$ & $\begin{array}{l}\text { No se puede obviar la incidencia de ciertos } \\
\text { factores individuales relacionados con la } \\
\text { subjetividad y la atribución de sentido a la propia } \\
\text { situación de precariedad, vulnerabilidad o } \\
\text { exclusión. }\end{array}$ \\
$\begin{array}{l}\text { Tiene un factor subjetivo/ } \\
\text { individual/personal }\end{array}$ & \\
\hline
\end{tabular}

Fuente: Hernández (2010). 


\section{Tabla 3. Comunalidades México y Costa Rica}

\section{Extracción}

MX CR

1. La utilización de las TIC al incorporarse al nivel universitario permite superar las condiciones de pobreza de los estudiantes en vulnerabilidad social.

2. El acceso a Internet desde su centro educativo permite establecer la permanencia de los estudiantes en la carrera que cursa.

3. El uso de las herramientas interactivas que provee Internet (Web 2.0 y 3.0) le ofrece valor agregado a su educación evitando la exclusión del centro educativo.

4. El acceso a Internet en el centro educativo permite mejores relaciones entre los compañeros.

5. El acceso a internet le permite acceder a recursos digitales que de otra manera no podría obtener.

6. El uso de las TIC propicia una relación en favor de la igualdad entre estudiantes.

7. La utilización de repositorios y bases permiten estudiar temáticas de índole social en relación con su carrera.

8. El uso de Internet genera una mejor comunicación con compañeros que utilizan este medio.

9. La utilización de las TIC ayuda a un mejor desarrollo de los contenidos impartidos por sus docentes.

$.217 \quad .628$

10. En el uso de las TIC como herramienta para el aprendizaje está enfocado en las necesidades educativas y sociales de los estudiantes.

11. Las TIC promueven espacios para el análisis sobre la problemática social en su comunidad.

12. El uso de las TIC propicia el trabajo colaborativo en las tareas académicas de los estudiantes.

13. Los estudiantes excluyen a sus compañeros si no utilizan TIC.

14. La utilización de las TIC permite mantener un trato igualitario entre los participantes.

15. La utilización de las TIC genera acciones que repercuten positivamente en el estudiantado.

16. La utilización de las TIC permite mantener un punto de vista crítico desde la perspectiva que generan las desigualdades sociales en el centro educativo.

17. El uso de las TIC puede generar un proceso que ayude a los estudiantes a disminuir la realidad desigual que experimenta.

18. La utilización de las TIC permite una mejor interacción entre estudiantes y profesores.

19. La utilización de las TIC permite generar transformaciones educativas y sociales de los estudiantes.

20. La utilización de las TIC permite evitar la discriminación social entre los estudiantes.

21. La utilización de las TIC genera mayor oportunidad educativa a los estudiantes.

22. La utilización de las TIC permite integrar socialmente a estudiantes de estratos socioeconómicos bajos.

23. El uso de las herramientas gratuitas que provee Internet le ofrece valor agregado a su educación evitando la exclusión del centro educativo en el que estudia.

Método de extracción: análisis de componentes principales.

Fuente: elaboración propia (2019). 
Tabla 4. Varianza explicada México

\begin{tabular}{|c|c|c|c|}
\hline \multirow{2}{*}{ Componente } & \multicolumn{3}{|c|}{ Autovalores iniciales } \\
\hline & Total & \% de varianza & $\%$ acumulado \\
\hline 1 & 4.858 & 21.123 & 21.123 \\
\hline 2 & 2.377 & 10.334 & 31.457 \\
\hline 3 & 1.739 & 7.560 & 39.017 \\
\hline 4 & 1.430 & 6.216 & 45.232 \\
\hline 5 & 1.306 & 5.678 & 50.910 \\
\hline 6 & 1.139 & 4.952 & 55.862 \\
\hline 7 & 1.125 & 4.890 & 60.752 \\
\hline 8 & 1.003 & 4.360 & 65.112 \\
\hline 9 & .987 & 4.293 & 69.405 \\
\hline 10 & .828 & 3.599 & 73.004 \\
\hline 11 & .772 & 3.357 & 76.361 \\
\hline 12 & .764 & 3.320 & 79.681 \\
\hline 13 & .695 & 3.022 & 82.704 \\
\hline 14 & .645 & 2.806 & 85.509 \\
\hline 15 & .547 & 2.377 & 87.886 \\
\hline 16 & .491 & 2.135 & 90.021 \\
\hline 17 & .469 & 2.038 & 92.059 \\
\hline 18 & .459 & 1.998 & 94.056 \\
\hline 19 & .386 & 1.680 & 95.736 \\
\hline 20 & .314 & 1.366 & 97.103 \\
\hline 21 & .265 & 1.151 & 98.254 \\
\hline 22 & .235 & 1.023 & 99.277 \\
\hline 23 & .166 & .723 & 100.000 \\
\hline
\end{tabular}

Fuente: elaboración propia (2019) 
Tabla 5. Varianza explicada Costa Rica

\begin{tabular}{|c|c|c|c|}
\hline \multirow{2}{*}{ Componente } & \multicolumn{3}{|c|}{ Autovalores iniciales } \\
\hline & Total & \% de varianza & $\%$ acumulado \\
\hline 1 & 7.967 & 34.641 & 34.641 \\
\hline 2 & 2.202 & 9.572 & 44.213 \\
\hline 3 & 1.907 & 8.293 & 52.506 \\
\hline 4 & 1.342 & 5.835 & 58.341 \\
\hline 5 & 1.135 & 4.937 & 63.278 \\
\hline 6 & 1.066 & 4.634 & 67.912 \\
\hline 7 & .916 & 3.982 & 71.894 \\
\hline 8 & .801 & 3.483 & 75.378 \\
\hline 9 & .694 & 3.017 & 78.394 \\
\hline 10 & .677 & 2.944 & 81.338 \\
\hline 11 & .636 & 2.766 & 84.105 \\
\hline 12 & .589 & 2.562 & 86.667 \\
\hline 13 & .501 & 2.178 & 88.845 \\
\hline 14 & .454 & 1.976 & 90.821 \\
\hline 15 & .381 & 1.658 & 92.479 \\
\hline 16 & .337 & 1.466 & 93.945 \\
\hline 17 & .296 & 1.287 & 95.233 \\
\hline 18 & .260 & 1.129 & 96.361 \\
\hline 19 & .250 & 1.089 & 97.450 \\
\hline 20 & .185 & .804 & 98.253 \\
\hline 21 & .178 & .774 & 99.027 \\
\hline 22 & .120 & .523 & 99.550 \\
\hline 23 & .103 & .450 & 100.000 \\
\hline
\end{tabular}

Fuente: elaboración propia (2019). 
Tabla 6. Matriz de patrón para México

\section{Ítems}

La utilización de las TIC al incorporarse al nivel universitario permite superar las condiciones de pobreza de los estudiantes en vulnerabilidad social.

El acceso a Internet desde su centro educativo permite establecer la permanencia de los estudiantes en la carrera que cursa.

El uso de las herramientas interactivas que provee Internet (Web 2.0 y 3.0) le ofrece valor agregado a su educación evitando la exclusión del centro educativo.

El acceso a Internet en el centro educativo permite mejores relaciones entre los compañeros.

El acceso a internet le permite acceder a recursos digitales que de otra manera no podría obtener.

El uso de las TIC propicia una relación en favor de la igualdad entre estudiantes.

La utilización de repositorios y bases permiten estudiar temáticas de índole social en relación con su carrera.

El uso de Internet genera una mejor comunicación con compañeros que utilizan este medio.

La utilización de las TIC ayuda a un mejor desarrollo de los contenidos impartidos por sus docentes.

En el uso de las TIC como herramienta para el aprendizaje está enfocado en las necesidades educativas y sociales de los estudiantes.

Las TIC promueven espacios para el análisis sobre la problemática social en su comunidad.

El uso de las TIC propicia el trabajo colaborativo en las tareas académicas de los estudiantes.

Los estudiantes excluyen a sus compañeros si no utilizan TIC.

La utilización de las TIC permite mantener un trato igualitario entre los participantes.

La utilización de las TIC genera acciones que repercuten positivamente en el estudiantado.

La utilización de las TIC permite mantener un punto de vista crítico desde la perspectiva que generan las desigualdades sociales en el centro educativo.

El uso de las TIC puede generar un proceso que ayude a los estudiantes a disminuir la realidad desigual que experimenta.

La utilización de las TIC permite una mejor interacción entre estudiantes y profesores.

La utilización de las TIC permite generar transformaciones educativas y sociales de los estudiantes.

La utilización de las TIC permite evitar la discriminación social entre los estudiantes.

La utilización de las TIC genera mayor oportunidad educativa a los estudiantes.

La utilización de las TIC permite integrar socialmente a estudiantes de estratos socioeconómicos bajos.

El uso de las herramientas gratuitas que provee Internet le ofrece valor agregado a su educación evitando la exclusión del centro educativo en el que estudia.

\section{Componentes}

1

2

$.033 \quad .518$

$-.025$

.468

.496

.174

$.178 \quad .294$

$.402 \quad .161$

$.221 \quad .256$

$.433 \quad-.208$

$.481 \quad .179$

$.487 \quad-.124$

$.546 \quad-.065$

$.479 \quad .145$

$\begin{array}{ll}.596 & -.088\end{array}$

\begin{tabular}{ll}
$.391 \quad .595$ \\
\hline
\end{tabular}

$.219 \quad .562$

$.455 \quad .170$

$-.072 \quad .587$

$.336 \quad .416$

$.652-.155$

$\begin{array}{ll}.756 & -189\end{array}$

$\begin{array}{ll}-.130 & .701\end{array}$

$.625 \quad .166$

$-.027 \quad .624$
$\quad .627$

.449

Método de extracción: análisis de componentes principales.

Método de rotación: Promax con normalización Kaiser.

La rotación ha convergido en tres iteraciones.

Fuente: elaboración propia (2019) 